opherre für offerre geschrieben habe; camisia und braca sind nicht germanisch (S. 83) sondern gallisch; ital. boccale bedeutet nicht nur Flasche (S. 107) oder besser Krug, sondern auch Pokal; poussade hat nichts mit passade (S. 108) zu thun, sondern ein Mädchen poussieren ist etwa Abänderung eines franz. pousser de beaux sentiments; dass die welschen Wörter, die mit $g$ oder gu anfangen, fast alle deutsch seien (S. 110) ist ein arger Schnitzer; Staket gehört nicht $\mathrm{zu}$ ital. steccato (S.111) sondern zu staken mit romanischer Endung; was hat Zerbino (S. 112) mit Zierbengel zu thun? was Belisar (S.113) mit dem weissen Zar? Graf ist natürlich nicht rpaqtúc und mandarin mit portug. mandar 'befehlen' gänzlich unverwandt; über Schwager für Postillon (S. 122) s. das richtige bei Kluge Deutsche Studentenspr. S. 15; dass die Griechen die Katze jemals als katol-

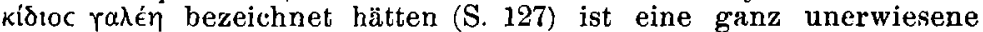
Behauptung; dass der indische Jagdleopard, der gepard, den deutschen Namen Gebhard führe (S. 128) ist liebenswürdig aber unwahrscheinlich ausgeklügelt; bei der Erklärung; von Bronze (s. 137) ist die Unüberlegtheit wohl auf Seite des Autors; diemant erscheint schon bei W. v. d. Vogelweide usw. usw.

Man thäte aber Unrecht deswegen das Büchlein ungelesen aus der Hand zu legen. Es ist das Werk eines krassen Dilettanten, aber doch eines geistreichen Menschen. Man sehe sich nur die Kapitelüberschriften daraufhin an oder kluge Bemerkungen wie die über den Unterschied der Geschlechter im Fremdwörterbesitze (S. 13) oder über romanische Ausdrücke als Übersetzungen deutscher (S. 38; vgl. Duvau Mèm. de la soc. de ling. 8, $184 \mathrm{ff}$.). Als Teil der für weite Kreise bestimmten Sammlung Göschen wird es freilich weit mehr Schaden als Nutzen stiften.

Bern.

S. Singer.

\title{
Mitteilungen.
}

\section{Bericht über die Verhandlungen des XII. internationalen Orientalistenkongresses in Rom, 3. bis 15. Oktober 1899.}

Vorbemerkung des Berichterstatters.

Während der Dauer des Kongresses habe ich nicht daran gedacht, über die dabei gepflogenen Verbandlungen für irgend eine Zeitschrift Bericht abzustatten, und ich habe mir in Folge dessen auch keinerlei Aufzeichnungen gemacht. Gleichwohl meinte ich, als mich ein Brief des Herausgebers dieser Zeitschrift vom 2. 11. 99 zur Berichterstattung aufforderte, nicht ablehnen zu sollen, von der freilich anfechtbaren Ansicht ausgehend, dass es immer noch besser ist Lückenhaftes als gar nichts vom Kongress zu hören. Es karnen Fragen aus dem Gebiet dieser Zeitschrift zur Besprechung in den allgemeinen Sitzungen, ferner in den Sitzungen der linguistischen, indischen, iranischen und der griechisch-orientalischen Sektion. Die der letztgenannten Sektion habe ich nicht besucht, kann also auch 
über die darin gehaltenen Vorträge nicht berichten ${ }^{1}$ ). - Ich bitte den Leser, diese Vorbemerkungen im Auge behalten zu wollen. Den Herren Vortragenden, die mich auf meine Bitte unterstützt haben, sei auch an dieser Stelle bestens gedankt. Die mir zugestellten, wörtlich abgedruckten Berichte sind durch *** gekennzeichnet.

\section{Linguistischë Sektion.}

Als Präsidenten fungierten: Ascoli, Bartholomae, Henry, Ludwig und Thomsen, als Sekretäre Goidanich und Boisacq. J. Leumann, Strassburg" sprach "zur indischen Sprachgeschichte".**** Auf Grund von alten Palmblatthandschriften wird gezeigt, dass bis etwa zum 7. Jahrhundert nach Chr. Geb. ziemlich allgemein in Indien nicht das korrekte Sanskrit, sondern verschiedene, mehr oder weniger ungenaue und durch die Dialekte beeinflusste Abarten des Sanskrit geschrieben wurden. Nur ein recht beschränkter Kreis von wohlgeschulten Brahmanen vermochte das pedantische Sanskrit, wie es die Grammatiker formuliert hatten, vollkommen zu bemeistern. Alle übrigen Schriftsteller, Brahmanen, Buddhisten und Sinisten schreiben das Sanskrit so gut sie es eben bei ihrer Bildung fertig brachten. Erst mit der Kommentatorenzeit, in der Samkara und Haribhadra hervorragen, hob sich der Standard des Sanskrit in weiteren Kreisen, und manches, was früher erlaubt gewesen war, wurde nun vermieden. Auch wurden die Unkorrektheiten älterer Texte sowohl von Kommentatoren als von gebildeten Abschreibern nunmehr gründlich ausgemärzt, so dass uns gegenwärtig meist nur noch solche Texte, von denen uns recht alte Handschriften erhalten sind, lehren können, wie nachlässig einst die subtilen Regeln der Sanskritgrammatik befolgt worden sind und wie das eigentlich lebendige Sanskrit ohne Rücksicht auf jene künstlichen Regeln unaufhaltsam seinen sprachlichen Entwicklungsgang durchgemacht hat.

In einem bekannten Sprichwort (das auch in Pischels Sanskritgrammatik S.53 steht) hiess es ursprünglich nirujasya, mit $\mathrm{z}$ wei Anomalien:

1) $n i r^{\circ}$ statt $n i r^{\circ}$, also ohne die durch die Grammatik geforderte Dehnung,

2) ${ }^{\circ} j a s y a$ statt ${ }^{\circ} j a h$, indem $n \bar{\imath} r u j$ - wie hundert andre konsonantisch-schliessende Komposita in die $a$-Deklination überführt wurde. Nur eine geringe Anzahl dieser Überführungen wurde von Grammatikern und Lexikographen approbiert, die Lexika erwähnen z. B. bloss nirruj. und ignorieren das allein von alters her übliche nīruja-.

V. Henry, Paris verbreitete sich über eine eigentümliche Art der Infigierung im heutigen Dialekt von Kolmar im Ober-Elsass. "Les formes visées sont les conditionals $i$ vęstit "je saurais", $i$ khentit 'je pourrais', $i$ miestikt ou miestit "je devrais "etc. etc." So nach einer brieflichen Mitteilung des Herrn Redners, der im Übrigen auf sein Buch "Dialcete Alaman de Colmar (Hte Alsace)" verweist, das in nicht allzulanger Frist erscheinen soll.

Gius. Ciardi-Dupré, Florenz trug vor "sul trattamento delle liquide primitive indoeuropee nell' indoiranicos. $0^{\circ * *}$ Wie Fortunatov KZ. 36, 1 ff. und Wackernagel Ai. Gr. 1, 217 nehme auch

1) Vgl. jetzt Krumbachers Bericht "Die mittel- und neugriechische Philologie auf dem Orientalistenkongress in Rom", Byz. Ztschr. 9, $312 \mathrm{ff}$. 
ich an, das idg. $l$ sei mundartlich im Altindischen unverändert geblieben, jedoch nicht aus denselben Gründen, von denen jene Gelehrten ausgehen. Dem erstgenannten gilt die Bewahrung des $l$ in einem Teil des indischen Sprachgebiets als eine notwendige Bedingung für das Wirken des von ihm BB. 6, 215 ff. angedeuteten Gesetzes, dass ich mit Bartholomae und $\mathrm{J}$. Schmidt fürr ganz falsch ansehe. Auch was Wackernagel a. a. O. vorbringt, überzeugt mich nicht. Der bündigste Beweis für die Bewahrung des idg. $l$ im Indischen wird meines Erachtens von der überwiegenden Zahl der Beispiele für ind. $l=$ idg. $l$ geliefert, im Vergleich mit denen für ind. $l=$ idg. $r$. Der Kern meiner Annahme liegt in der Zählung und Prüfung der in Betracht kommenden Wörter. Der Beispiele - von aind. $l$ - für idg. $l$ sind es nicht weniger als 130 , jener für idog. $r$ etwa 20-25. Eine solche Ungleichheit kann keine zufällige sein. Die Entwicklung der Liquiden im Indischen lässt sich in folgender Weise begreifen. Im Urarischen erhielt sich die ursprüngliche Unterscheidung beider Liquiden, und diese lebte in einer indischen Dialektgruppe fort, während in einer andern $l$ mit $r$ zusammenfiel. $\mathrm{Zu}$ dieser letztern gehört das Vedische, zu jener der Grundstock der klassischen Sprache. Das Vorkommen von Formen mit $l=\mathrm{idg}$. $l$ im Vedischen und mit $r=\mathrm{idg} . ~ l$ im Sanskrit beruht wohl auf Dialektmischung, und, was die zweite Erscheinung anbelangt, auf dem von den Veden auf die spätere Sprache ausgeübten Einfluss. Daneben gab es auch Dialekte (die als Vorstufe des sog. Mittelindischen anzusehen sisd), darin $r$ vorhistorisch zu $l$ geworden war. Aus einem solchen Dialekt werden die vedischen und klassischen Wörter stammen, die ein $l=\mathrm{idg} . r$ enthalten. - Das Iranische betrachte ich nur anhangsweise. Die älteren uns bekannten Vertreter dieser Familie zeigen den Wandel von $l$ zu $r$, aber für die neueren iranischen Sprachen, namentlich fürs Neupersische (das vom Altpersischen in verschiedener Hinsicht abweicht) fehlt es nicht an Gründen anzunehmen, oder genauer gesagt $\mathrm{zu}$ vermuten, es sei das $l$ mundartlich auch hier geblieben. Also ist das Zusammenfliessen der idg. $r, l$ in $r$ kein allgemeines Kennzeichen des arischen Sprachzweiges, da es in einem Teile des indischen und vielleicht auch des iranischen Gebiets nicht stattgefunden hat. Ob zwischen dem indischen und dem iranischen Rhotazismus ein historischer Zusammenhang besteht, darüber kann man bloss Vermutungen aufstellen.

P. G. Goidanich, Napoli ${ }^{1}$ ) bespricht ${ }^{s i}$ modi nelle sintassi delle lingue indoeuropee". **** L'ottativo nelle sintassi dell' ario e del greco ha due funzioni: esprime una mozione volitiva ("modus optativus', 'modus praescriptivus') ed è il 'modus potentialis'. - Le due funzioni sono fra loro psicologicamente irreducibili, nessuna delle due puó esser dall' altra derivata. Come pertanto venne alla stessa forma la duplice funzione? La risposta è assai facile a darsi. L'indicativo e l'imperativo hanno il tema indentico: ciò mostra che gl' Indoeuropei non ebbero due forme per distinguere l'ennunziazione dall' ingiunzione che pur sono due categorie psicologiche essenzialmente distinte. Ora l'optativus potentialis, psicologicamente appartiene alla categoria psicologica dell' enunziazione, come l'indicativo; con la differenze che l'indicativo serve ad enunziare un fatto od uno stato reale e l'ottativo serve ad enunziare alcunché in maniera potenziale. L'ottativo del desiderio appartiene, come

1) Nunmehr Pisa. 
l'imperativo alla categoria psicologica dell' ingiunzione (infatti, i continuatori di i. e. *bhéroim(i), *bhérois, *bheroit mai significano: "ich wünsche zu tragen, .. dass du trägst, .. dass er trägt" [Delbrück SF. 1], ma: 'possa io, tu, egli portare'; e nessun argomento abbiamo per credere che in tempo preistorico sia stato diversamente); con la differenza che l'inperativo appartiene alla categoria psicologica della realtà e l'ottativo esprime invece un volere della cui effettuazione il parlante non è certo, un volere dunque espresso in maniera potenziale. Essenziale è dunque come nel tema di indicativo-imperativo la nota della realtà, cosi nel tema dell' ottativo enunziativo e desiderativo la nota della potenzialitá, accidentale è invece la funzione enunziativa o ingiuntiva anche nell' ottativo, come tutti ammettono che sia accidentale nel tema di indicativo-imperativo.

Il congiuntivo (prescindendo per il momento dall' innegabile sua affinità col tema del futuro) ha due funzioni esso pure, una enunziativa ed una ingiuntiva. Anche 'ingiuntiva' ho detto, perchè in tutte le persone meno che nella prima il congiuntivo ha valore esortativo; e la prima non può esser critica (Hopkins Am. J. of Phil. 13, 34). Anche qui nessuna delle due funzioni pù esser la fondamentale per irreducibilità psicologica. Invece anche nel congiuntivo troviamo che nota comune tanto nella funzione ingiuntiva quanto nella funzione enunziativa è la potenzialità. La potenzialità è dunque anche qui la nota caratteristica essenziale, l'ingiunzione e l'enmunziazione sono funzion $i$ accidentali, come nell' indicativo-imperativo e nell' ottativo optativus-potentialis.

Meno il greco tutte le altre lingue hanno o mostrano di aver avuto un sincretismo sintattico tra congiuntivo e ottativo. Nel greco

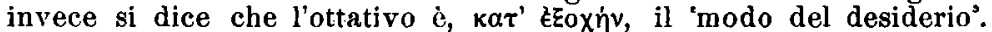
Ora si può facilmente osservare che tra 'esortazione' e 'desiderio che son le funzioni ingiuntive del congiuntivo e dell' ottativo vì è una differenza nel grado della potenzialità. Ma questa stessa differenza che troviamo nella categoria ingiunzione esiste tra congiuntivo e ottativo anche in funzione ennunziativa; le frasi enunziative

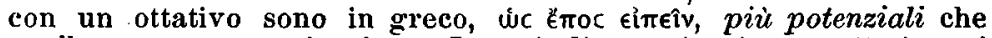
quelle con un congiuntivo. I temi di congiuntivo e ottativo si distinguono dunque in greco per il grado della potenzialità. In nessuna altra lingua manifestamente ${ }^{1}$ ) nè una tale nè qualsivoglia altra differenza tra congiuntivo ad ottativo esiste; ma, posto pure che il greco continui condizioni indoeuropee l'esponente differenziale fra i due modi è sifattamente piccola ${ }^{2}$ ) che si resta meravigliati a pensare come mai due modi siano etimologicamente diversi e si è indotti invece a sospettare che almeno un medesimo principio morfologico abbia sopraseduto alla genesi die entrambi $\mathrm{i}$ temi di modo. Per dimostrar questo quesito io segno la via seguente.

Il tema del congiuntivo si distingue da quello dell' indicativo par la maggior ricchezea di una mora all esito. Ora $i$ temi in vocale lunga invariabile (Brugmann $\mathrm{Cl} \mathrm{X}$ e XI) e in parte quelli in vocale lunga variabile (Brugmann Cl. I, III, V, XII) non erano in grado di formare un congiuntivo con questo sistema. Ma un

1) Jo trovo traccie di ottativi nal congiuntivo italico in -sem; come non potrei qui brevemente accennare.

2) Cf. anche Whitney (Am. J. Phil. 13, 294) a proposito della differenza tra i concetti desiderio e volontà e Delbrück Vgl. Synt. 2,351 . 
mezzo per ovviare a questo ostacolo vi era. Accanto a temi di qualsivoglia classe corrono paralleli temi in $j e / i$; accanto a temi $\bar{a}^{x}$, corrono temi paralleli in $(\breve{a} x)+i e, \bar{a}^{x}+i$ (Brugmann $\mathrm{Cl}$. XXVI e XXVII, Bartholomae Stud. II); questi si che potevano fare un congiuntivo, cioè di fronte ad un indicativo $(-\not{\partial} x) \cdot i e$ un congiuntivo $(\breve{d} x) \cdot i \bar{e}$, di fronte ad un indicativo $-\bar{a}^{x}(\cdot i)$ un congiuntivo $-\bar{a}^{x} \cdot i e$ (confr. Joî $\mu \in v$

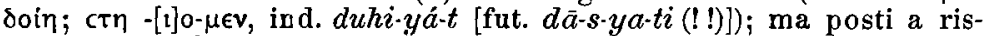
contro di indicativi-imperativi in $-\bar{a}^{x_{-}}$, simili congiuntivi dovevano far sembrare quale caratteristica della potenzialità $i \check{e}$, che è la caratteristica dell ottativo!

Tra congiuntivo e futuro esiste un affinita morfologica incontrastabile. Si dice ordinariamente che il congiuntivo venga adoperato per il futuro. Ma questo procedimento nelle fasi storiche inandito ed psicologicamente ingiustificabile. Dalle fasi storiche invece apprendiamo il procedimento opposto e ben giustificabile, che per l'espressione della potenzialità vengano assunte forme di futuro (o anche di passato). Io reputo quindi che un tale procedimento psicologico sia da ritenersi, data la coircidenza morfologica tra congiuntivo e futuro, avvenuto anche in periode proetnico i. e. -

S'indende, dopo ció, che il processo di segmentazione sopra notata non sara da reputarsi avvenuto tra forme indicativali e forme della potenzialità, ma prima tra presente e future. In una fase più vicina a noi il futuro (e il passato ${ }^{1}$ ) saranno stati assunti come espressioni della potenzialità; il suffisso $\ddot{i} \breve{~ p o i ~ s i ~ r i s t r i n s e ~ i n ~ p i u ̀ ~ a n-~}$ gusti confini non rimanendo nella categoria primitiva, nella categoria del futuro, altro che nel futuro in -s-ie-, mentre le forme caratterizzate con $\breve{e} / \breve{o}$ ed $\vec{a}^{x}$ restarano tanto per la espulsione del futuro che per quella della potenzialita (congiuntivo).

Chr. Bartholomae (Giessen) gibt kurze Mitteilungen über Umfang und Anlage seines altiranischen Wörterbuchs, dabei die Hoffnung aussprechend, sein Werk dem nächsten Kongress fertig vorlegen zu können.

P. Karolides (Athen) trägt über die phrygisch abgefassten Inschriften Kleinasiens vor. Er sucht zu erweisen, dass ihre richtige Deutung durch Vergleich mit dem Armenischen zu finden sei. Als Beleg führt er aus der Midas-Inschrift das Wort síkeman an, das er als ñoûov oder 'königliches Grab' deutet und mit dem armen. sigai in Zusammenhang bringt.

Giac. de Gregorio (Palermo) handelt "sugli elementi orientali nelle lingua neolatine". Wenn man, so führt Redner aus, die Dialekte des Südens genauer studiere, so finde man, dass der Einfluss des Arabischen hier weit stärker sich geltend gemacht habe, als etwa Körting's Wörterbuch vermuten lasse. Gegenüber der Annahme baskischer Einflüsse auf die romanischen Sprachen verhält sich Redner ablehnend. Inschriften".

A. Torp (Christiania) sprach "zur Erklärung der lykischen

W. Thomsen (Kopenhagen) brachte mit einigen begleitenden Bemerkungen zwei Druckschriften zur Verteilung: "Etudes lyciennes 1 " und "Remarques sur la parenté de l'étrusque"; nach der letzteren "la parenté de l'étrusque devrait être recherchée dans les langues du Caucase, et plus spécialement dans celles du Nord ou langes lesghiennes".

1) Alludo al greco col quale hanno alcuni ricontri il gotico e il latino: ma qui par la brevità dello spazio non postrei esprimermi con la voluta chiarezza. 


\section{I. Indische Sektion.}

Präsidenten: Hoernle, W. Hunter, Kuhn, Pischel, Sénart, R. West; Sekretäre: Formichi, Kirste, Thomas.

J. Kirste (Graz) bringt seinen Vorschlag über die Einordnung des Anusvāra und Visarga im Sanskritalfabet zur Sprache, wie er den Sanskritisten bereits durch Umschrift bekannt gemacht worden war. In der Diskussíon sprach sich die Mehrzahl der Redner für die Beibehaltung der durch das Petersburger Wörterbuch sanktionierten Ordnung aus.

V. Henry (Paris) bespricht die Rigvedazeile 1. 152. 2 c: triráśrim hanti cáturaśrir ugró. Grammatisch sei sie einfach genug, aber ihr Sinn sei nicht leicht $z u$ fassen; habe doch $I_{\Delta} \mathrm{u} d$ wi $\mathrm{g}^{*}$ nicht weniger als drei, allerdings leichte Korrekturen vornehmen müssen ${ }^{1}$ ) um zu einer Deutung zu gelangen. Redner fasst, ohne den überlieferten Text zu ändern, die Zeile als ein naturalistisches Rïtsel: "der vier Spitzen hat schlägt den nit drei Spitzen" oder anders ausgedrückt "das Dreieck wird von dem Viereck zu Nichte gemacht". Letzteres, das Viereck, beziehe sich auf die Somme, die nach den Brāhmanaś catuhsrakti- sei, ersteres auf das letzte Viertel des Monds, das von der Sonne eingeholt und verschlungen werde.

E. Le u man n (Strassburg) macht Mitteilungen ïber den Kranz von Erzählungen, die sich an den Namen des Königs Brahmadatta von Kampilya knüpfen und in fünf Gruppen zerfallen: Brahmadatta als cakravartin-, Br. und seine sechs Wiedergeburtsgenossen, Sünde von Br.s Sohn und ihre Sühne, Jugendabenteuer Br.s bis dass er cakravartin- wird, Br.s Speisung der Bahmanen.

E. Hardy (Würzburg) spricht über die beiden unter den Namen Petavatthu- und Vimänavatthu-gehenden Erbauungsbücher des alten Buddhismus. Litterarisch ohne besondere Bedeutung seien sie doch von Wert als Proben volkstümlicher und alter, wennschon unter dem Einfluss der fortschreitenden Zivilisation umgemodelter Erzählungen. Etwa im 3. Jahrhundert vor Chr. müssten sie bereits zu kanonischer Geltung gelangt sein.

Gerson da Cunha (Bombay) handelt über die Rāmatanka, d. i. hohlrunde Medaillen, geprägt zur Erinnerung an die Krönung der Könige von Vijāyanāgara in Südindien und schon seit mehr als einem halben Jahrtausend als köstliche glückbringende Reliquien geschätzt und verehrt. Auf der Innenseite zeigen die Münzen eine Darstellung der Krönung Rāmas - daher ihr Name und seiner Gattin Sītā.

Fräulein E. Plunket trägt über Astronomie im Veda vor. Die Thatsache, dass die zwölf Sternbilder des Tierkreises den Westasiaten bereits 4000 Jahre vor Chr. bekannt waren, erlaube die Annahme, dass auch die Brahmanen schon lange vor der griechischen Invasion mit der Teilung des Zodiacus vertraut gewesen seien. Darauf sich stützend könne man vier wichtige vedische Mythen erklären: 1) Indra sei der Gott des Sommersolstizes, der den Vrtra vertreibe, d. i. die Konstellation Hydra; 2) Soma pavamāna repräsentiere den Vollmond des Sommersolstizes, grereinigt in den Wassern der Konstellation Aquarius; 3) Agni sei das Feuer der Sonne beim Wintersolstiz, in der Konstellation Aquarius; 4) die Aśvin seien auf die Sterne des Naksatra Ašvini zu beziehen, die durch ihren helischen Aufgang die Wiederkehr des neuen Jahrs anzeigten. In

1) Ludwig wollte: trír áśrir hanti cátur ásrir ugrá (oder ugró) lesen. 
der sich anknüpfenden Debatte bleiben Kern (Leyden) und Jacobi (Bonn) dabei stehen, es seien die astronomischen Kenntnisse den Indern von den Griechen zugebracht worden. Pischel (Halle) verweist auf Thibauts baldigst $z u$ erwartende Geschichte der indischen Astronomie.

C. Formichi (Bologna) bespricht den Kãmandakīyanītisāra. Man könne seine Entstehung nicht früher setzen als ins fïnfte oder sechste Jahrhundert nach Chr. Das zeigten deutlich zwei Stellen des Textes, die die Bekanntschaft des Verfassers mit der Philosophie des Nyäya und anderseits astronomische Anschauungen bekundeten, die denen Varähamihira's nahe stehen.

E. La Terza (Castro Giovanni) versucht zu zeigen, dass der Dichter Bhartrhari dem fünften Jahrhundert nach Chr. angehöre und nicht ein Buddhist, sondern ein Vedāntin gewesen sei. Der Verfasser der S'alakaś werde auf das Zeugnis des Chinesen Itsing hin mit dem des Väkyapadīya verwechselt.

Fr. Hewitt (London) sprach über "The history of the ark or ship of the gods, its astronomical origin and later forms ${ }^{33}, R$. Temple (London) verlas eine Abhandlung R. Fleets "Curiosities of Indian epigraphy", Brajendranath Seal (Cooch Behar) über indische Philosophie, P. Deussen (Kiel) über die Geschichte der Philosophie der Upanisad's. W. Radloff (Petersburg) berichtet über Handschriften-, Bücher- und Inschriftenfunde in Turfan, C. Bendall (Cambridge) über solche in Nepal; R. Hoernle (Oxford) legt eine ausserordentlich interessante Sammlung zentralasiatischer Altertümer vor: Manuskripte, Holzschnitte, Medaillen usw., darunter Handschriften aus dem 4. und 5. Jahrh. nach Chr.

\section{Iranische Sektion.}

Präsidenten: Browne, Esow, Geiger, Jackson, Salemann; Sekretär: Ciardi-Dupré.

Will. Jackson (New York) berichtet über den Plan eines awestischen Wörterbuchs, das er zu schreiben vorhabe; W. Geiger (Erlangen) über neuerliche Vermehrung unserer Kenntnisse der iranischen Dialekte des asiatischen Hochlands; Fr. Poinetti (Rom) spricht über "le relazioni diplomatiche tra la Santa Sede e la Persia" und über die Dokunente, die über jene Beziehungen Aufschluss geben.

\section{Allgemeine Sitzungen.}

Präsident: de Gubernatis, Ehrenpräsident: Ascoli, Sekretär: Pullé.

Conte Fr. Pulle (Pisa) macht Mitteilung von seinem Plan das auf Indien bezügliche kartographische Material der italienischen Archive und Bibliotheken zu sammeln und herauszugeben. Füntzig: in Buntdruck hergestellte Karten veranschaulichen die geographischen Anschauungen über Indien zu verschiedenen Zeiten und bei den verschiedenen Völkern.

M. Macauliffe (London) handelt "on the life and writings of guru Gobind Singh, the tenth guru of the Sikhs", wobei er in Kürze auch auf die Religion der Sikhs eingeht. In der sich anschliessenden Debatte wird eine Übersetzung der heiligen Bücher der Sikhs für dringend wünschenswert erklärt.

Brajendranath Seal (Cooch Behar) verliest eine Abhand-

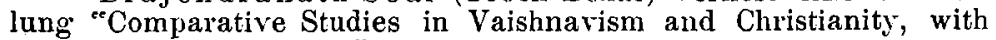
their historical relations". 
Fr. Gamurrini spricht über die vor kurzem auf dem Forum ausgegrabene alte Säule, den niger lapis, von dem das Unterrichtsministerium dem Kongress einen Gipsabguss zur Verfügung gestellt hat. Alles deute darauf hin, dass unter dem niger lapis ein Heroon gewesen sei ${ }^{e c}$ che per tradizione riferita da Varrone si designava per il sepolero di Romolo".

Zum Schluss sei noch erwähnt, dass während des Kongresses eine "Association internationale pour l'exploration archéologique de l'Inde" begründet wurde, und dass E. Kuhn und L. Scherman mit dem Plan einer "Indischen Biblographie" hervorgetreten sind.

Giessen.

Bartholomae.

\section{Zu dem 'Vorwort' zu Band 1 der Morphologisehen Untersuchungen von Osthoff und Brugmann.}

Man lasse sichs gefallen, dass ich an dieser Stelle etwas nachhole, was ich schon vor 21 Jahren hätte thun können uud sollen. Zugleich, dass ich über einen damals in Kurs gekommenen Ausdruck eine Aufklärung gebe, die, wenn nicht aus einem andern Gesichtspunkt, so doch vielleicht als ein Scherflein zur Geschichte moderner Witz- und Spitznamen veröffentlichungswert ist.

In seiner jüngst erschienenen tüchtigen Arbeit 'Giebt es Lautgesetze?", Halle 1900 (Sonderabzug aus der Festgabe für Hermann Suchier), schildert Eduard Wechssler den 'Kannf um die Lautlehre', der nach 1870 in der indogermanischen Sprachwissenschaft entbrannte. Dabei wird unter anderm jenes in der Überschrift genannte, vom Juni 1878 datierte und gemeinschaftlich von Osthoff und mir unterzeichnete längere 'Vorwort', das ja in den Debatten über die methodischen Grundsätze unserer Disziplin eine Rolle gespielt hat, angozogen, aber Osthoff wird von Wechssler als alleiniger Verfasser desselben angesehen: s. S. 75 und S. 152. Ich vermutete, dass diese Ausschliessung meiner Person von der Autorschaft den nemlichen Grund habe wie zwei oder drei gleiche Ausschliessungen, die mir in früheren Jahren zu teil geworden sind, und eine von Herin Wechssler auf Anfrage gütigst mir erteilte Auskunft ergab die Richtigkeit dieser Vermutung. Ein Rezensent der Morph. Unt. hatte nemlich im Jahr 1879 im Anzeiger für deutsch. Altertum 5, S. 318 sich also vernehmen lassen: "Ich sage 'd ie Verfasser der Vorrede'. Vielleicht würde man richtiger sagen 'd er. Verfasser'. Denn das Vorwort ist, nach dem 'Tone und nach gewissen Eigenheiten im Ausdruck zu schliessen, allein von Osthoff verfasst. Unterzeichnet aber ist es von beiden Verfassern, und deshalb haben beide die Verantwortung zu tragen für die Haltung: und den Inhalt desselben". Wechssler hat, wie er mir schreibt, diesem Rezensenten vertrauen $z u$ können geglaubt, durch ihn sei er zu seiner Angabe bestimmt worden, die er von sich aus nicht. gemacht hätte. Was es mit den 'gewissen Eigenheiten im Ausdruck' auf sich hat, die den Scharfsinn des Kritikus geleitet haben, mag dieser mit sich selber ausmachen. Ich aber möchte denn doch, um der Legendenbildung nicht länger Vorschub zu leisten, beute erklären, dass das Vorwort nicht von Osthoff, sondern von mir verfasst worden ist. Ich schickte seinerzeit das Manuskript zur Begutachtung an Osthoff, und, soviel ich mich entsinne and Osthoft sich entsinnt, hat dieser sich darauf beschränkt, eine oder zwei 